\title{
Model of Interoperability of Multiple Different Information Systems using SOA Middleware Layer and Ontological Database on the Cloud
}

\author{
Meryem FAKHOURI AMR, Khalifa MANSOURI, Mohammed QBADOU, Bouchaib RIYAMI \\ Laboratory: Signals, Distributed Systems and Artificial Intelligence (SSDIA) \\ ENSET Mohammedia, Hassan II University of Casablanca, Morocco
}

\begin{abstract}
The exponential evolution of technology and the environment surrounding the information systems (IS) forces companies to act quickly to follow the trend of business workflows with the use of high computer technologies and well adapted to the needs of the market. Currently, the performance of information system is considered a problem, we must intervene to make them more agile and responsive to better support the strategy of the company. The exchange and communication is a must between information systems to address these issues and new requirements, businesses are looking to integrate and interact even their information systems to interconnect applications. Interoperability is essential between information systems, it promotes alignment between the company's business strategy and IT strategy while respecting the existing technical heritage of the company. The interoperability solutions between information systems face major problems since the SI is independently developed and designed differently and that solutions must meet certain criteria, namely, autonomy, scalability and resolve trade problems of data. The MDA approach is the most suitable solution to our problem because it ensures a degree of independence between the logic the company's business and technology platform. Moreover, oriented architecture SOA service is used in this sense, it encapsulates the components of the information system into editable and reusable service. We want through this article to contribute to the development of a model for interoperability of several different IS, founded on a middleware layer compound of services according to the architecture SOA. The special feature of our model is that it uses an ontological database in the Cloud that will store concepts exchanged among interconnected IS and uses several transformation layers, integration, homogenization and adapting services.
\end{abstract}

Keywords-Interoperability; middleware layer; SOA; information system (IS); cloud computing; ontological database $(O D B)$

\section{INTRODUCTION}

The information system is the nerve center of any organization because it allows it to share information with other IS and collaborates. Therefore, interoperability between information systems is crucial since the applications should be adapted in order to determine for each request, the relevant data sources, the required syntax for querying the terminology specific to the source, to combine fragments of the results from each source to construct the final result [1].
Today, the major concern of every business is to develop integrated applications to solve the interoperability problem. The increasing complexity of information systems has created a critical need for models and methods of reuse. Companies are obliged to deal with this problem by evolving their information systems and adapting their business strategies to the technical strategy [2]. In this paper, we provide a model of the interoperability which is going to interface several information systems which are different by founding itself on services oriented architecture SOA. This model affords an SOA middleware layer that eliminates the complexity of interconnected IS and makes them homogeneous for interoperability, this model also uses other conversion and adaptation layers. The new contribution of this paper is that it is based on the use of an ontological database will be hosted on the Cloud. The organization of this article will follow the following order. We start by introducing the main work on the study of interoperability between information systems and the role of architecture led by MDA models and the implementation of SOA at the IS to ensure better communication. We also present the different approaches used to design this model of interoperability as a state of the art, we explain the SOA middleware layer that presents our contribution. We go on to describe the layers that make up the model by describing it by a schema. We conclude by summarizing the main contributions and highlighting future work.

\section{RELATED WORK}

Today the world is suffering from strong competition and from the uncertainty of markets. This turbulent change has led companies to rapidly evolve to keep this wave of transformation of enterprise information systems and ensure better interoperability between them. Several studies have focused on interoperability between information systems. According to Agostinho, [3] interoperability, is the best solution to the problem, forcing companies to adapt and organize themselves to an automated information exchange. $\mathrm{He}$ strongly believes in the dynamism of interoperability, this is the solution that counts for the future; however, it considers that the static interfaces that exist today are no longer valid for tomorrow, and the solution is to maintain interoperability networked information systems. He acknowledged that before, there was an unassailable gap between the requirements of companies and the implementers of information systems. But with the implementation of architectures led by MDA models 
and interoperability model-driven, the concept of integrated design attenuated this gap.

To overcome this problem of complexity of the software development process, integration of new application and interoperability, Ameedeen [4] proposes a method of interoperability models using model-driven development to bridge the difference between the design and analysis phases of software development. This method consists of a diagram of transition sequences to Petri nets.

Despite the rapid development of technology and the evolution of information systems, there are still few methodologies that have been adopted to facilitate the development of software and interoperability between them. Loukis [5] admitted that the implementation of the interoperability of the information systems of a company with those of its employees generates commercial value, though this point has been studied in a limited way.

Today, companies are moving towards service-oriented architectures seen its many benefits, although it is not easy to talk about the interoperability of business processes in an environment oriented service. To solve this problem faced by most businesses, Tebib [6] proposes the use of interaction protocols (IP) as an effective means to structure and organize the exchange of messages between partners. For this, she used the BPEL4WS language that is considered a language of Web services composition for the definition of business processes and description of interactions between services.

The interoperability of information systems is changing especially with the use of SOA services oriented architectures for IT systems involved. This combination of technologies related to ontologies, service models ensures better semantic interoperability of services provided and requested [7]. According to Jiucheng [7], the purpose of business is to bring their heterogeneous IS and ensure that critical applications work perfectly. An approach based on a model including semantic annotation service models with ontologies can support and enhance semantic interoperability in serviceoriented systems. We cannot deny that in recent times, the development of models for the interoperability of information systems using SOA Service-oriented architecture has gained immense importance. Batra has a concise proposal in this area [8]. He proposed a system that uses the SOA-compliant web services concept, which involves exchanging provider data and transforming it into a standard data exchange format using two databases. Both databases can be in different system formats. So, it is necessary to convert them to standard format prior to storage in the data repository. The whole of the process is implemented using Web services and messages are exchanged using the SOAP protocol.

It is in this sense that our contribution fits, which involves the implementation of model of interfacing and adaptation based on MDA models and SOA middleware layer for interconnection of several different systems information. This model is founded on a number of business process transformation layer BPMN towards the language BPEL using an ATL execution language by using an ontology database hosted in the Cloud.

\section{StATE OF THE ART: APPROACHES TO MODEL CONCEPTION}

\section{A. Approach MDA (Model Driven Architecture)}

The field of interoperability promotes the use of the MDA approach because it ensures the introduction of isolation between the business layer and the implementation layer. This approach is part of a field of engineering models driven by IDM (IDM or MDE Model Driven Engineering) models. This approach takes models into account and focuses development on them [6].

The importance of the MDA approach is the development of systems, it is oriented model because it provides the basis for the use of models to orient and guide the understanding, design, construction, deployment, maintenance and modification of the systems developed. MDA primary objectives are portability, interoperability and reusability [7].

The motivation behind the use of this approach is that it can meet the major needs of task reduction to re-design applications that have become a technological development, a requirement for companies. Since the design of the models is unchangeable, the codes, with the implementation of the MDA approach, will allow to keep the business requirements, to use the code, to ensure the integrity and coherence.

\section{B. Service Oriented Architecture SOA}

SOA is a functional architecture that provides the ability to structure functional services in relation to current technologies. SOA is used to ensure the integration of business applications [8]. The characteristic of SOA architecture is that they allow to separate the specification of the art treatments interfaces on the one hand, and to propose composition approaches for constructing process by assembling services on the other hand. Based on a set of de facto standards, the implementation of an SOA can meet, at least in technology, the problem of interoperability of IS components [8].

This approach allows the encapsulation of applications into services which is a reusable and detached software entity during its use [9]. This operation named the composition of application services or more technically, the orchestration of web services [8]. A web service is designed as a software program, its role is to guarantee the interconnection and the sharing of data between different distributed applications [8].

\section{Intermediate Approach}

Mediators are used as a generic model of mediation that ensures the interconnection between the IS [10]. Mediators represent themselves according to two categories that can be executed in order to fulfill their main mission of mediation [11], [12].

We intend to use the fundamentals of the MDA approach with great benefits, focusing on models as well as their designs compared to conventional programming [13].

The need for using the MDA approach appears in the conversion of BPMN to the language BPEL (Business Process Execution Language) of different IS interconnected. Later, we design a common executable BPEL language that includes the BPEL (Business Process Execution Language) languages of 
the interconnected IS. The differences will be dealt with using an ontological database. To create this language, we use the intermediary approach that will allow us from the resulting BPEL Global language, to transform it into a business process BPMN (Business Process Model and Notation) thanks to the retro-engineering.

\section{SOA MIDDLEWARE LAYER FOR INTEROPERABILITY OF MULTIPLE IS}

The upward approach is the method we used to design the model. This approach starts from a base to arrive at a complete model. Our added value through this article is the design of the SOA middleware layer that will make the IS interoperable and reduce the complexity of communication and sharing between them. The major principle on which is based is the encapsulation of each part in a service.

The model we have designed consists of a number of conversion and adaptation layers. The interconnection takes place between $\mathrm{N}$ information systems. The major rule of our model is that it can interface any information system whatever the number of interconnected IS. The principle of this model is based on the composition of business processes and encapsulation services to facilitate modeling and reuse through the SOA middleware layer. Through this model, we want to promote integrated communication via information technologies as a single entity that is based on the SOA architecture.

\section{LAYERS CONSTITUENTS THE MODEL OF INTERFACING AND ADAPTATION FOR THE INTEROPERABILITY OF SEVERAL SIFFERENT IS}

The proposed model as presented in Fig. 1 is based on MDA architectures and intermediates as well as SOA. It consists of several layers:

Layer 1: This layer transforms BPMN business processes into an executable BPEL language by using an ATL (Atlas Transformation Language) transformation language from MDA, and we want this conversion to make systems agile and consistent

Layer 2: From this layer, the common BPEL languages are gathered from the interconnected IS BPMNs. the ontological database is used to treat the differences between the BPELs obtained. The ontological database we use is hosted in a public cloud. The use of ontologies provides a formal description of concepts in a domain and guarantees reuse, knowledge sharing and interoperability.

Layer 3: It is the time of the correction and the adaptation of the common languages obtained towards the end of the transformation of BPMN business processes into the BPEL languages of each IS, an update is required of the source business processes of the interconnected IS to synchronize all the BPMNs. To do this, we use reverse engineering, which allows us, through transformation techniques and rules, to have raw models from their BPEL source code [14].

\section{INTEROPERABILITY MODEL OF SEVERAL IS USING SOA SERVICE-ORIENTED ARCHITECTURE AND ONTOLOGICAL DATABASE ON THE CLOUD}

For the design of this model, we used the service-oriented SOA architecture to break business processes into services to facilitate their reuse or replacement by another service. The model makes it possible to communicate $\mathrm{N}$ System of information whatever the number of IS. In case the number of SI is odd, we gather the last SI with the last BPEL obtained. Our model will undergo some operations to arrive at generating the common global BPEL and transform it into a global BPMN that will help us, using an ontological database, to update the initial business processes BPMN of the interconnected IS.

We start by transforming the BPMN business processes from the first two ISs into the executable BPEL language using an ATL executable language. Subsequently, we bring the two BPEL together to a BPEL 1 Global. The differences that exist between BPELs are analyzed by a single ontological database. the same workflow is repeated for the other IS by bringing together the BPEL Global 1 obtained from the IS (1 and 2) with the BPEL Global 2 of the other IS until a cascading global model is obtained, which brings together the Global BPEL of all IS to have towards the end a single common GLOBAL BPEL. Thanks to the reverse engineering, we transform the BPEL GLOBAL language into a GLOBAL BPMN business process that will allow us to apply an update for all the source BPMN to ensure a better synchronization of the IT trades.

The model we proposed for the interoperability of multiple different IS characterized by a Cloud architecture that will allow it to store data exchanged between interconnected IS. The ontological database shared between the IS will be put into service on the Cloud is also the source of sharing and the communication link between the interconnected IS. The particularity of this architecture of an ontological database on the Cloud, is that it is more practical than the other architectures and presents many advantages for the implementation of our model, namely that all the concepts exchanged between the IS interconnected will be stored in this ontological database, the difference between all the obtained Global BPEL will be processed through this ontological database hosted on the Cloud. This architecture promotes the sharing and availability of data located at the level of the ontological database between all the interconnected IS as well as its rapid deployment and its simplicity of integration in addition to its high availability, since the ontological database will be on the Cloud which will guarantee the IS a better accessibility to resources.

The motivation behind the establishment of the ontological database on the Cloud is justified by the fact that this new architecture has many advantages in that the database will be maintained and usually stored on a secure server, it also allows ease of remote access to the ontological database or even a simple deployment of the database if a new information system will interconnect with other IS. 


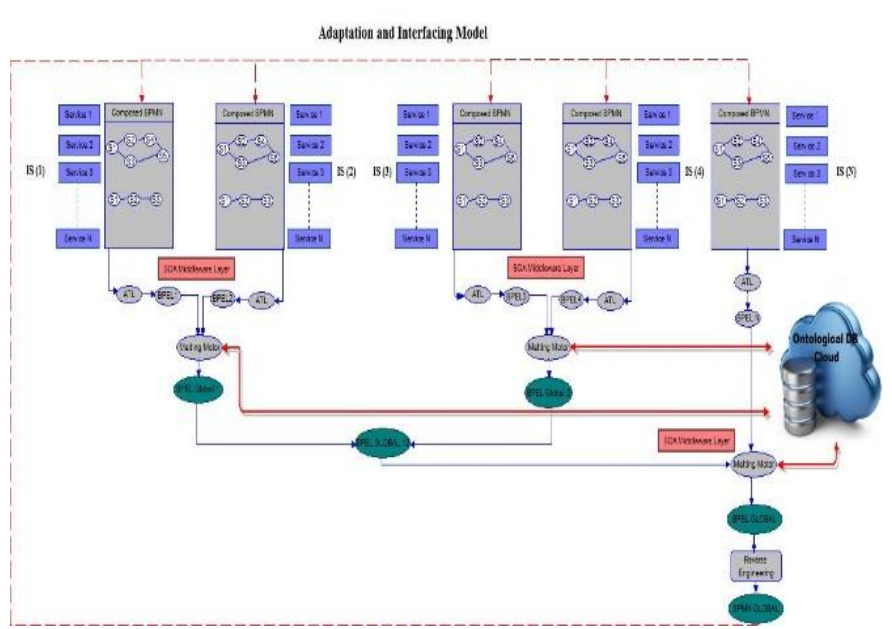

Fig. 1. Interworking and adaptation model for interoperability of multiple ISs using a SOA middleware layer and cloud database.

SOA is an approach that allows the management of information system components of a company as reusable services on this basis our interoperability model has been developed. Our solution is based on a middleware layer that presents the central and vital element for our SOA architecture, which will facilitate exchange and interoperability between information systems interconnected by integrating a database in a Cloud space. The establishment of a Cloud architecture facilitates easy access to computing resources by users of a company, the total availability of resources on the Internet and payment for the use of these resources.

Given the advantages offered by these two architectures, SOA and Cloud, it was thought to combine the two architectures to give information systems of interconnected companies more agility, ease of sharing and communication. It will also reduce the complexity of systems through the reuse of services and of course the optimization of resources. The model that we propose allows an easy communication between the information systems, it guarantees a high availability of the ontological database with a very high security.

\section{Cloud ARCHITECTURE ADOPTED FOR THE PROPOSED MODEL FOR INTEROPERABILITY OF IS}

Today's markets need cloud computing which has had to situations in exponential growth. But its definition remains general especially that few people agree on a specific definition. Cloud means "applications delivered as Internet services, typically network, hardware and software systems in the data centers that provide these services" [15]. Cloud Computing allows any user to use multiple computer forums for a period of time to perform calculations, process data, or create Web applications [15].

Defined as a new technology, Cloud Computing allows the implementation of IT services through the management and use of information systems. Despite the difficulties through the use of Cloud expressed by the lack of standards and heterogeneous architectures, there is a compelling solution for migrating to new environments [16].
The choice of Cloud Computing for the implementation of our model was not random because the Cloud presents to the field of interoperability of information systems, several advantages [16], we quote:

- Reducing infrastructure costs: Information systems interconnected via this model must have a specific technical architecture to be able to interoperate with other IS which generates high costs. So, the proposed solution will guarantee IS the use of a shared Cloud infrastructure between them without the need for an internal architecture.

- Reduced development costs: The model deployed for interoperability will be centralized between IS interconnected which will generate a single centralized development task. All that comes under the maintenance of the technical infrastructure is supported by the provider of Cloud solution.

- Reduce software costs: Our model is based on an architecture based on a single ontological database so it will allow companies to save software costs used for its implementation and the costs associated with Cloud architecture.

- Ease of access to shared resources and services: This is the major advantage of the Cloud architecture, it facilitates an easy, secure and safe for even resources with the combination of SOA, there will be no difficulty in sharing services.

- Increased processing power of shared data: Cloud architecture with the data processing will be easier because this architecture has a set of powerful technical components in terms of capacity, strength and speed.

Cloud computing can be deployed according deployment models that have been treated and quoted by several studies. The four deployment models:

- Public Cloud: The peculiarity of the public Cloud is that it is open to the public, the infrastructure is managed by the provider who takes care to offer its services to users. All computing resources are shared among users who have no control or visibility into infrastructure [16].

- Private Cloud: The Private Cloud hosting infrastructure is controlled and in the ownership of the user of the resources. These are available and intended for private use.

- Hybrid Cloud: It's an environment that combines both models Public and Private. Resources are allocated from a private Cloud and a public Cloud.

- Cloud Community: The Community Cloud infrastructure is designed to use a very specific community of users. This infrastructure can be managed by one or more community organizations [16].

The model we propose for the interoperability of information systems involves a Cloud infrastructure to host the 
ontological database and computer technologies deployed for the implementation of this model between the information systems of interconnected companies. The choice of this infrastructure is justified by the fact that it has many advantages especially as information and shared data between the IS must be centralized and accessible by all IS interconnected.

Since the establishment of a specific architecture for each company wishing to interoperate with another, is too expensive and consuming IT resources, the solution we propose is to put this model on a Hybrid Cloud infrastructure that will allow users to share private data on the private Cloud and public data on the public Cloud, in this way all users will have equal access to shared resources.

\section{SERVICES COMPONENT THE MODEL OF INTEROPERABILITY}

\section{A. BPMN-BPEL Transformation Service}

The BPMN business process is used to model the company's business into a workflow that describes the system's functionalities and makes it possible to understand it in detail. This workflow can be used and shared with other systems. BPMN is designed by the Business Process Management Initiative (BPMNI) and managed by the Object Management Group (OMG) [17]. While the BPEL model is a programming language developed to handle orchestration processes [17].

The MDA approach ensures the process of transforming BPMN into BPEL through service transformation. You have to go from a conceptual model to an executable source code. To succeed this process, we must not go directly from one model to another but to go through their meta-models. The MDA approach ensures the process of transforming BPMN into BPEL through service transformation. You have to go from a conceptual model to an executable source code. To succeed in this process, we must not go directly from one model to another but to go through their meta-models. Typically, the processing service supports models (sources) and model outputs (target). Transformation allows us to gain a lot of development time by generating an automated code [17].

We start the model process by converting each BPMN business process into a BPEL executable code. We have presented the business process by its abstraction model which is a subset of information of this system. We speak in our case, meta-models that describe the general model, it defines the elements that constitute it, its overall structure and its semantics. We cannot proceed to the manipulation of the metamodel if the language that describes it is not ready [18]. In our case, we are talking about a meta-meta-model. Meta-metamodels are characterized by their self-defining capabilities, which is due to the principle of the Model Driven Engineering (MDE) approach [18]. There are some actions to put in place on the input models to have corresponding output models. It brings us to two categories of transformations, from models to models and models to text. As it is mentioned in Fig. 2, in our case, we are interested in the transformation of the model to the text, which allows us to transform the BPMN business process to a BPEL code and to gather all the BPEL code and generate a
BPEL GLOBAL code. ATL (Atlas Transformation Language) is a language based on the definition of metamodel, its principle is the transition from one model to another via metamodels [17].

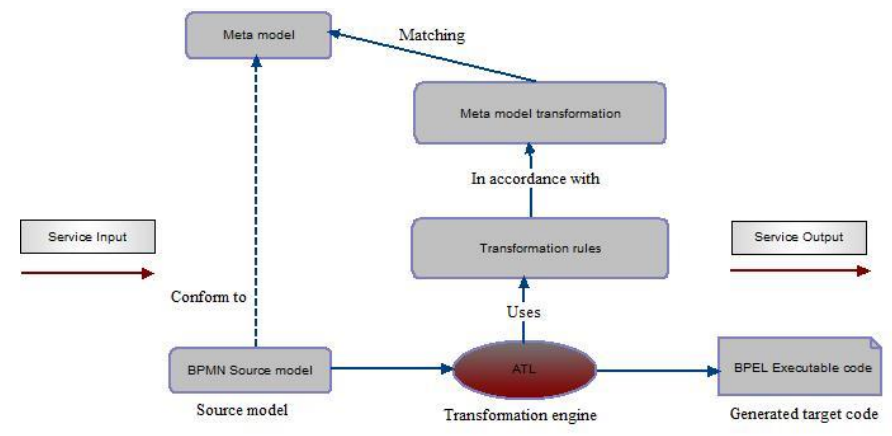

Fig. 2. BPMN to BPEL transformation service according to MDA [19].

\section{B. Consolidation, Homogenization and Integration Service}

The consolidation service provides a BPEL that contains different items and others in common. We do a BPEL analysis obtained by the homogenization and integration service, then bring the two BPELs into a common BPEL containing common elements and differences. These differences between BPEL will be analyzed by an ontological database hosted on the Cloud as it is described in Fig. 3. The consolidation, homogenization and integration service accepts the BPEL code as input and generates a BPEL executable code.

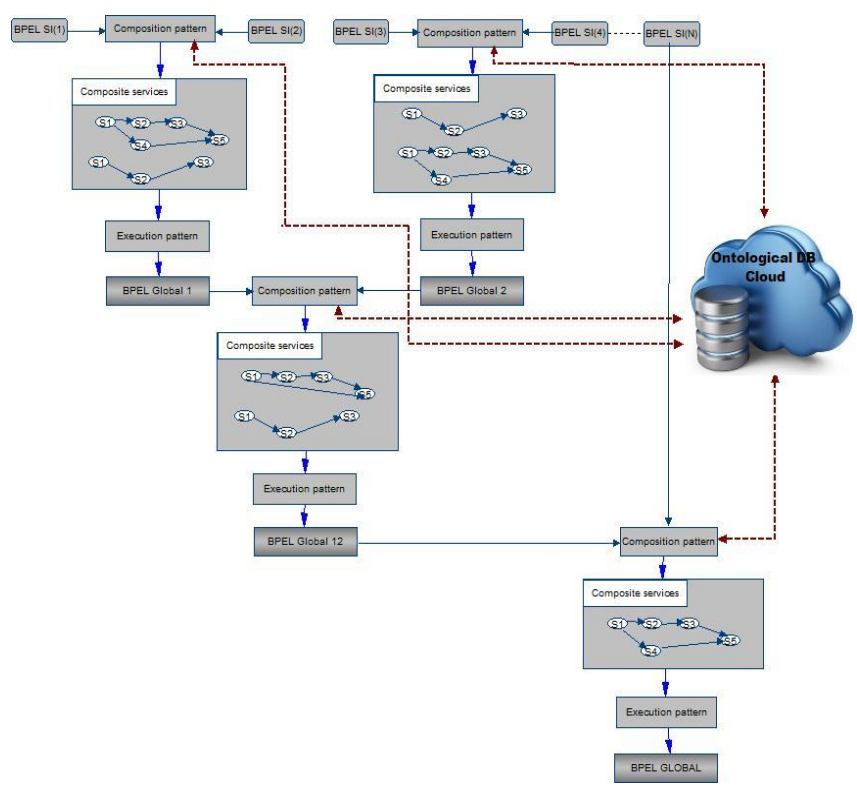

Fig. 3. Process for obtaining a GLOBAL BPEL using an ODB on Cloud.

Ontology is a conceptualization that makes it possible to represent the semantics of a domain. For this purpose, she uses models of consensual objects that associate each concept with an identifier [20]. The ontological database we use is hosted on the cloud. It makes it possible to treat the differences between the BPEL obtained [21]. Ontologies allow their reuse, so have other concepts of the domain which will ensure the interconnection and exchange of data sources [21]. 


\section{C. "Reverse Engineering" Service}

Thanks to the retro engineering and its techniques and rules as represented in Fig. 4, we will make an adaptation of the BPEL languages obtained. At the end of the BPMN business process transformation into a BPEL code, we assign an update to the source business workflows of the information systems to synchronize the Global BPMN. It is about managing the knowledge to answer the technical and functional specifications at the origin of the initial model, then to structure, formalize and update them [22].

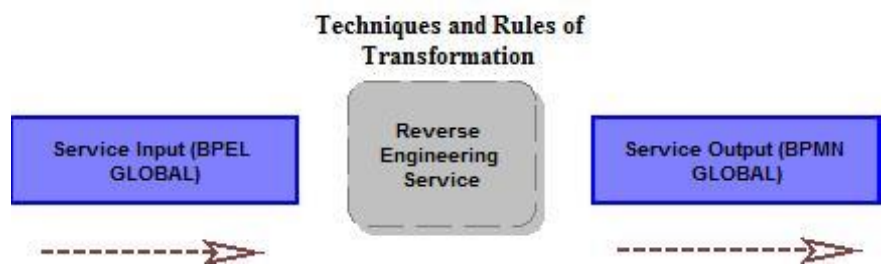

Fig. 4. "Reverse Engineering" Service [19].

\section{CONCLUSION}

In this article, we developed a model for interoperability of multiple IS that remains valid regardless of the nature of the interconnected information system and its field of activity. This model can interoperate multiple information systems based on the SOA middleware layer. This model contains layers of transformation, consolidation, homogenization and integration of services. Throughout the development of this model, several approaches have been used in view of the advantages they present, including MDA, intermediate approach (ontologies) and the SOA approach for encapsulation of each IS component in a service. Towards the end reverse engineering was used, it was used for reconstitution and the updating of BPMN business process of each information system after the transformation and adaptation. The peculiarity of our contribution is that we used the ontological databases to treat the difference between BPEL obtained through the union of BPEL of interconnected SI by hosting this ontological database on the Cloud to benefit from its many advantages. In perspective, we consider the implementation of this model to interconnect different hospital information systems, also based on aspects of the SOA approach given its ability to optimize and improve business processes.

\section{REFERENCES}

[1] J. Moskolai, A.Ayissi R. Hoe-Ngouna, Integration and interoperability of heterogeneous information systems in distributed environments.. Towards a flexible approach based on the urbanization of information systems, International Research Conference computer, Dec 2013, Yaounde, Cameroon. 2013.

[2] S. Izza, Integration of Industrial Information Systems: a flexible approach is based semantic services. Modeling and simulation. Ecole des Mines de Saint-Etienne, 2006. French
[3] C. Agostinho, Y. Ducq, G. Zacharewicz, J. Sarraipa, F. Lampathaki, Towards a sustainable interoperability in networked enterprise information systems: Trends of knowledge and model-driven technology, Computers in Industry, Elsevier, 2016, Future Perspectives On Next Generation Enterprise Information Systems, 79, pp.64 - 76.

[4] M.A. Ameedeen, B. Bordbar, R. Anane, Model interoperability via Model Driven Development, Journal of Computer and System Sciences, Volume 77, Issue 2, March 2011, Pages 332-347.Elsevier, https://doi.org/10.1016/j.jcss.2010.01.011.

[5] J-M Jézéquel B. Combemale, D. Vojtisek Engineering Model Driven: concepts to practice, Ellipses. Ellipses, pp.144, 2012 References sciences 9782729871963

[6] M. RADGUI, decomposition and adaptive business processes BPMN for flexible information systems, in June 2015.

[7] A. Authosserre-Cavarero, F. Bertrand, F. Blay-Fornarino,O. Collet,D. Hu-bert, Interoperability of information systems: model-driven approaches, Inforsid, Montpellier, France. pp.11-30, 2012

[8] S. Baina, H. Panetto, K. Benali, The MDA approach Contribution to semantic interoperability: Interoperability of Enterprise Information Systems, Journal of Science and Information Technology -Series ISI. Systems Engineering Information Lavoisier, 2006, 11/13, pp.11-29

[9] K. Dahman, Governance and study the impact of changing business processes on service-oriented architectures. One approach model-driven, Hardware Architectures [cs.AR], University of Lorraine, 2012. French

[10] A. Zayati., Implementation of service-oriented architectures for industrial information systems. Business administration. INSA Lyon, 2012. French.

[11] M. Weske, Business Process Management: Concepts, Languages, Architectures. 2012 ,DOI 10.1007/978-3-642-28616-2 Springer

[12] P. Degoulet, L. Marin, L. Kleinebreil, B. Albiges, Present and future of information systems and hospital communication, Springer Paris, 2003.

[13] T. Erl, SOA: Principles of Service Design, USA: Prentice HALL PTR, 2007.

[14] I.Noe Garcia Garza, design and implementation models for mediation and integration services, Other [cs.OH] .University Grenoble, 2012. French

[15] M. Durut., PhD Thesis. Algorithms classfiication distributed cloud. Learning [cs.LG]. Telecom ParisTech, 2012. French

[16] H. Medhioub., Architectures and federation mechanisms in environments cloud computing and cloud networking. Other [cs.OH]. National Institute of Telecommunications, 2015, French.

[17] K. Mansouri, B. Riyami, M. Youssfi, O. Bouattane, MODEL OF AN ADAPTATION AND INTERFACING SOA MIDDLEWARE FOR THE INFORMATION SYSTEMS INTEROPERABILITY, JOURNAL OF THEORETICAL AND APPLIED INFORMATION TECHNOLOGY, FEBRUARY 2016.

[18] N. F. Noy, D.L. McGuinness, Ontology Development 101: A Guide to Creating Your First Ontology. Université Stanford

[19] M. FAKHOURI AMR, K. Mansouri, M. Qbadou, Towards a model of adaptation and interfacing based on a middleware layer SOA for interoperability of several different information systems., 2017 Morocco, IEEE Computer Society, The second International Conference on Intelligent Systems and Computer Vison (ISCV).

[20] A. kévin, A. Engel, Interoperability between models, Nancy, France, 2011.

[21] V. ARANEGA, Traceability to the development of models and correction of transformations, PhD Thesis, Graduate School: Engineering Sciences University lille1 November 2011, France.

[22] B.Mbaiossoum, S.Khouri, L.Bellatreche, S.Jean, M.Baron, Comparative Study based Database Systems Ontological, University of Poitiers. 